\title{
Unique structural features of a BCL-2 family protein CED-9 and biophysical characterization of CED-9/EGL-1 interactions
}

\author{
J-S Woo', J-S Jung ${ }^{1}$, N-C Ha ${ }^{1}$, J Shin ${ }^{2}$, K-H Kim ${ }^{3}$, W Lee ${ }^{2}$ and \\ B-H Oh*,1,3 \\ 1 Department of Life Science, Center for Biomolecular Recognition and Division \\ of Molecular and Life Science, Pohang University of Science and Technology, \\ Pohang, Kyungbuk 790-784, Korea; \\ 2 Department of Biochemistry, College of Science, Yonsei University, Seoul 120- \\ 749, Korea; \\ 3 Pohang Accelerator Laboratory, Pohang, Kyungbuk 790-784, Korea \\ * Corresponding author. B-H Oh, Department of Life Science, Center for \\ Biomolecular Recognition and Division of Molecular and Life Science, Pohang \\ University of Science and Technology, Pohang, Kyungbuk 790-784, Korea. \\ Tel.: + 82-54-279-2289; Fax: + 82-54-279-2199; \\ E-mail: bhoh@postech.ac.kr
}

Received 16.4.03; revised 06.6.03; accepted 10.6.03; published online 1 August 2003 Edited by $G$ Salvesen

\section{Abstract \\ The interactions between B-cell lymphoma 2 (BCL-2) family members are known to be mediated through the binding of the $\mathrm{BH} 3$ domain of a proapoptotic member to the BH3-binding groove of an antiapoptotic member. We determined the crystal structure of antiapoptotic CED-9, which reveals a unique C-terminal helix altering the common BH3-binding region. A coexpression system to produce CED-9 in complex with proapoptotic EGL-1 enabled us to show that the binding of EGL-1 to CED-9 is extremely stable, raising the melting temperature $\left(T_{\mathrm{M}}\right)$ of CED-9 by $25^{\circ} \mathrm{C}$, and that the binding surface of CED-9 extends beyond the BH3-binding region and reaches the BH4 domain. Consistently, the $T_{\mathrm{M}}$ and a ${ }^{1} \mathrm{H}-{ }^{15} \mathrm{~N}$ correlation NMR spectrum of CED-9 in complex with EGL-1 are drastically different from those of CED-9 in complex with the EGL-1 BH3 peptide. The data suggest that the recognition between other BCL-2 family members may also involve much wider protein surfaces than is previously thought. \\ Cell Death and Differentiation (2003) 10, 1310-1319, do:10.1038/ sj.cdd. 4401303 \\ Published online 1 August 2003}

Keywords: apoptosis; CED-9; EGL-1; BCL-2 family; molecular recognition

Abbreviations: $\mathrm{BCL}-2$, B-cell lymphoma 2; $\mathrm{BH}, \mathrm{BCL}-2$ homology; $C D$, circular dichroism; HSQC, heteronuclear single quantum; MAD, multiple-wavelength anomalous dispersion method

\section{Introduction}

B-cell lymphoma 2 (BCL-2) family proteins are the major regulators of apoptosis pathways. ${ }^{1}$ They are characterized by containing up to four conserved stretches of amino acids, designated as BCL-2 homology (BH) domains. Some members of this family are antiapoptotic and others are proapoptotic. The family can be divided into three subclasses according to the $\mathrm{BH}$ domain(s) that each class possesses. Antiapoptotic members, which include BCL-2 and BCL- $X_{L}$, belong to the subclass that contains all the four $\mathrm{BH}$ domains (BH1-4). Proapoptotic members can be classified into either the subclass that contains three or four $\mathrm{BH}$ domains $(\mathrm{BH} 1-3$ or $\mathrm{BH} 1-4$ depending on definition) or the subclass that contains only the $\mathrm{BH} 3$ domain. $\mathrm{BH} 3-$ only proteins share sequence homology in the $\mathrm{BH} 3$ domain, a 9-16 amino-acid segment, but are otherwise unrelated in the sequence and number of amino acids. Unlike the multi-BH domain proapoptotic members, the BH3-only proteins are held on check by transcriptional regulation or sequestration to cytoplasmic proteins. ${ }^{2}$ The interactions between the $\mathrm{BH} 3$-only members and the antiapoptotic members are crucial events in controlling or initiating apoptosis. ${ }^{3}$

In nematode Caenorhabditis elegans, the cell-killing process requires CED- 3 and CED- $4 .{ }^{4}$ CED- 3 is homologous to mammalian caspases, which, once activated, cleave various protein substrates in the dying cell. CED-4 is homologous to mammalian Apaf-1 and functions as a positive regulator of CED-3. ${ }^{5}$ The oligomerization of CED-4, like that of Apaf- 1 , is believed to activate CED-3 by bringing several inactive zymogen forms of CED-3 molecules into close proximity. ${ }^{6}$ CED-9 is the sole multidomain BCL-2 family member in the worm. CED- 9 prevents cell death by sequestering CED- 4 and the zymogen form of CED-3 in an inactive ternary complex. ${ }^{7-11}$ EGL-1 is the sole BH3-only protein in the worm and is transcriptionally regulated. The hallmark for all developmentally required apoptosis in $C$. elegans is the complex formation between CED-9 and EGL-1, which disrupts the CED-9 : CED4 complex, thereby releasing the CED- 4 molecule from the mitochondria into the perinuclear space, where it drives apoptosis. ${ }^{12,13}$ Conceivably, the interaction between CED-9 and CED-4 should be very strong, but the interaction between CED-9 and EGL-1 is much stronger than that.

Since a variety of $\mathrm{BH} 3$-only proteins and multi-BH domain proapoptotic proteins bind to the same antiapoptotic targets (e.g. BCL-2, BCL- $X_{L}, B C L-W$ ), the commonly present BH3 domain is believed to mediate the binding interactions. In support of this view, mutational analyses demonstrated that the $\mathrm{BH} 3$ domain of proapoptotic proteins is required for binding to antiapoptotic BCL-2 homologues and initiating apoptosis. ${ }^{14-16}$ The $\mathrm{BH} 3$ domain is known to bind a predominantly hydrophobic concave surface of a cognate partner known as the $\mathrm{BH} 3$-binding groove, as revealed by the 
solution structure of the complex between $B C L-X_{L}$ and the BAK BH3 peptide. ${ }^{17}$ However, it is not clear whether the $\mathrm{BH} 3$ domain and the $\mathrm{BH} 3$-binding groove are the only structural motifs that govern the binding specificity and affinity in the recognition between BCL-2 family members, and for this reason, in the mediation of apoptotic process. We show here that CED-9 and EGL-1 form an extremely stable complex, which behaves as if it is a single protein. The $2.03 \AA$ crystal structure of CED-9 (residues 68-251, C107S/C135A/C164S), site-directed mutagenesis, and physical analyses of the CED9:EGL-1 complex demonstrate that the EGL-1-recognition surface on CED-9 extends beyond the BH3-binding groove and reaches the $\mathrm{BH} 4$ domain. Herein, we also discuss a unique structural feature of a C-terminal region of CED-9.

\section{Results}

\section{Initial construct and modification of recombinant CED-9}

We generated a $C E D-9$ construct that contains residues 1-251 lacking the predicted C-terminal membrane-spanning region (residues 252-280). The nonconserved $\mathrm{N}$-terminal region (residues 1-67) of CED-9 contains two sites cleaved by CED-3 and was suggested to inhibit CED-3, probably through a competitive mechanism like that proposed for baculovirus protein $\mathrm{p} 35 .^{18}$ The $\mathrm{N}$-terminal region of the gene product appears unstructured, because it was easily degraded during the purification of the protein. The $\mathrm{N}$-terminal region is unlikely to contribute to the binding of CED-9 to EGL-1, because timecourse cleavage of CED-9 (residues 1-251) and the protein in complex with EGL-1 by caspase-3 did not show any difference in the cleavage patterns between the two (data not shown). Consistently, CED-9 (residues 68-280) was shown to interact with EGL- $1^{19}$ or CED- $4^{18}$ as fully as the full-length CED-9. Subsequently, we generated a CED-9 construct containing the $\mathrm{N}$-terminal deletion. Although the deletion mutant was expressed as a soluble protein, it precipitated easily during the protein purification and concentration. On a native polyacrylamide gel, we observed laddering of protein bands, indicating the formation of intermolecular disulfide bonds. Although the addition of the reducing agent dithiothreitol alleviated the problem, we could not obtain the crystals of the sample. Subsequently, we substituted all the three cysteine codons with serine or alanine codons, resulting in triple mutations of $\mathrm{C} 107 \mathrm{~S} / \mathrm{C} 135 \mathrm{~A} / \mathrm{C} 164 \mathrm{~S}$. These cysteine residues are not conserved and the residues corresponding to Cys107 and Cys164 are substituted with hydrophilic residues in several CED-9 homologues, indicating that they may be on the protein surface. The mutations eliminated the precipitation problem and the final protein sample was crystallized. Later, the CED-9 structure showed that Cys107 and Cys164 are located on the surface of the protein and Cys135 is a buried residue. In retrospect, Cys135 did not have to be changed.

\section{Overall structure of CED-9}

The crystal structure of CED-9 (residues 68-251, C107S/ C135A/C164S), which is referred to as CED-9 hereafter, was solved with the multiple-wavelength anomalous dispersion method (MAD) phasing, by using one crystal of selenomethionine-substituted CED-9. The resolution for the final model of CED-9 is $2.03 \AA$. The asymmetric unit of the crystal contained two molecules of CED-9. One molecule contains residues 71158 and 165-241, while the other molecule contains residues 75-242. The structure of CED-9 consists of seven $\alpha$-helices (Figure 1). Two central $\alpha$-helices $\alpha 5$ and $\alpha 6$ are surrounded by other $\alpha$-helices and loop $\alpha 1-\alpha 2$. The C-terminal helix $\alpha 7$ interacts mostly with $\alpha 2$. The CED-9 structure shares an identical folding topology with $B C L-X_{L},{ }^{20,21} B A X,{ }^{22}$ and $B C L-$ $W .^{23,24}$ The $\mathrm{BH} 4$ domain (residues 79-97) forms $\alpha 1$ and a part of loop $\alpha 1-\alpha 2$. The BH3 domain (residues 112-127) forms most of $\alpha 2$. The $\mathrm{BH} 1$ domain (residues 149-179) forms most of $\alpha 4$, loop $\alpha 4-\alpha 5$, and the first half of $\alpha 5$. The $\mathrm{BH} 2$ domain (residues 213-228) constitutes parts of $\alpha 6$ and $\alpha 7$. It should be noted that the lengths of the $\mathrm{BH} 1-4$ domains differ depending on researchers.

Loop $\alpha 1-\alpha 2$ in CED-9 is shorter and well defined (Figure 1b) compared with the corresponding loops in BCL-2 and BCL- $\mathrm{X}_{\mathrm{L}}$ that are significantly longer ( $\sim 58$ residues) and unstructured. ${ }^{20}$ The loop packs against both $\alpha 1$ and $\alpha 6$ by engaging in extensive hydrophobic interactions with the two helices (partly shown in Figure 1b). Loop $\alpha 1-\alpha 2$ in BCL-W is also short (Figure 2). Unlike in CED-9, the loop in BCL-W interacts with $\alpha 1$ and the $\mathrm{N}$-terminal part of $\alpha 2$, but not with $\alpha 6$. $^{23}$ As a consequence, the $\mathrm{BH} 4$ domain of CED-9 is less shielded than that of BCL-W. The accessibility to the BH4 domain of the antiapoptotic BCL-2 family members may bear a functional significance.

\section{Alteration of the BH3-binding groove}

The two most remarkable structural differences between CED-9 and the other known BCL-2-like protein are the conformation of the C-terminal segment and the shape of the $\mathrm{BH} 3$-binding region. The $\mathrm{C}$-terminal segment of CED-9 preceding the putative transmembrane region is longer than those of the other BCL-2-like molecules (Figure 1c). The segment interacts heavily with $\alpha 2$ (the $\mathrm{BH} 3$ domain) to form the amphipathic six-turn helix $\alpha 7$, which is considerably longer compared with the two-turn helix $\alpha 7$ in BCL- $X_{L}$ (Figure 2). The hydrophobic interactions between $\alpha 2$ and $\alpha 7$ contribute to the formation of a prominent hydrophobic groove below $\alpha 3$ and $\alpha 4$ (Figure 2c). While the substantial part of the BH3-binding groove in the other BCL-2-like molecules is formed by the wedge-like $\alpha 3$-loop- $\alpha 4$, the corresponding groove is absent in CED- 9 due to extensive interactions between the helices $\alpha 3$ and $\alpha 4$, that are parallel to each other. The presence of the long helix $\alpha 7$ and the different location of the hydrophobic groove render the $\mathrm{BH} 3-$ binding region of CED-9 drastically different from those of BCL-2, BCL- $X_{L}, B A X$, and BCL-W.

$A$ regulatory role of the $C$-terminal segment in the antiapoptotic BCL-2 family members has been suggested based on the BCL-W structure, which reveals that the $\mathrm{C}$ terminal region of the protein, like that of the proapoptotic protein $\mathrm{BAX},{ }^{22}$ occupies the BH3-binding groove. ${ }^{23,24}$ In the Bax structure, ${ }^{22}$ the groove-occupying residues are mostly hydrophobic residues, but in the structure of BCL-W, a hydrophilic stretch occupies the groove ${ }^{24}$ as well as the following hydrophobic tail. ${ }^{23}$ The structures of BCL-2 and 
$B C L-X_{L}$ were determined using truncated proteins that are devoid of the $\mathrm{C}$-terminal hydrophobic tail and a preceding short stretch. ${ }^{20,21,25}$ Therefore, whether the missing region may adopt the same conformation seen in the structure of BCL-W needs to be determined. In contrast, the C-terminal segment of CED-9, corresponding to the hydrophilic stretch of BCL-W, forms an extended amphipatic $\alpha$-helix that itself contributes to the formation of the prominent hydrophobic groove. When we expressed CED-9 containing the hydrophobic tail (residues 252-280) in Escherichia coli cells, the protein was totally insoluble. CED-9 localizes primarily to intracellular membranes and the perinuclear region in a manner that depends on the C-terminal hydrophobic tail. ${ }^{10}$ Most likely, the C-terminal tail of CED-9 is a transmembrane domain and would not bind to the BH3binding region of the protein in vivo. Since electron density for the C-terminal nine residues (residues 243-251) was not visible, this region appears to be a flexible linker between the putative transmembrane domain and the rest of the molecule. a

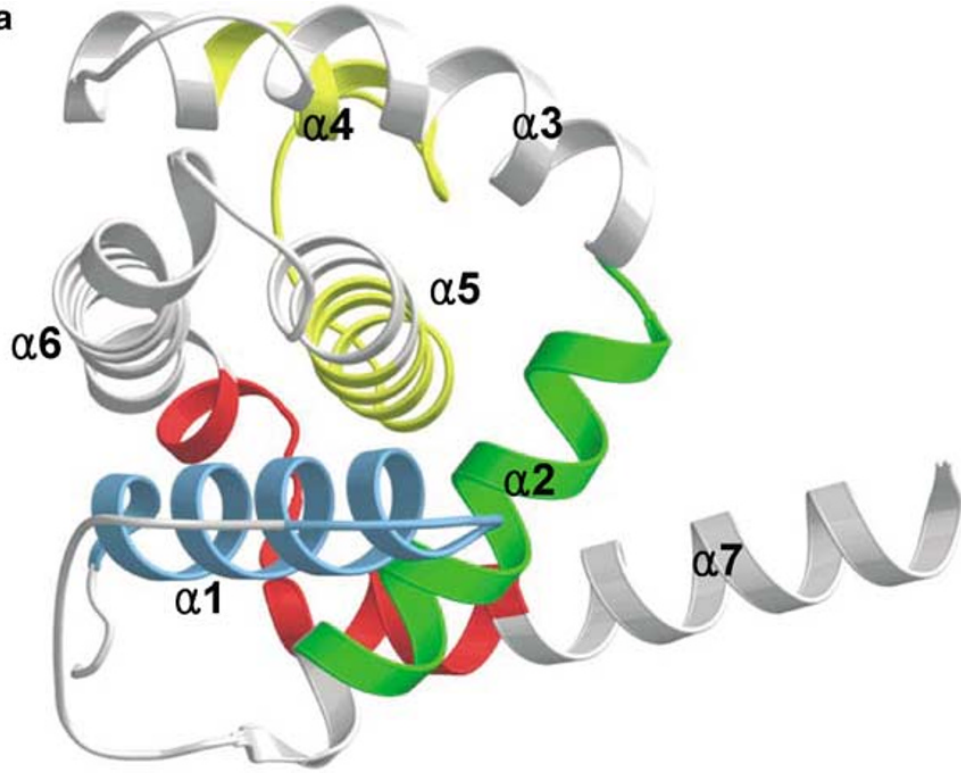

b

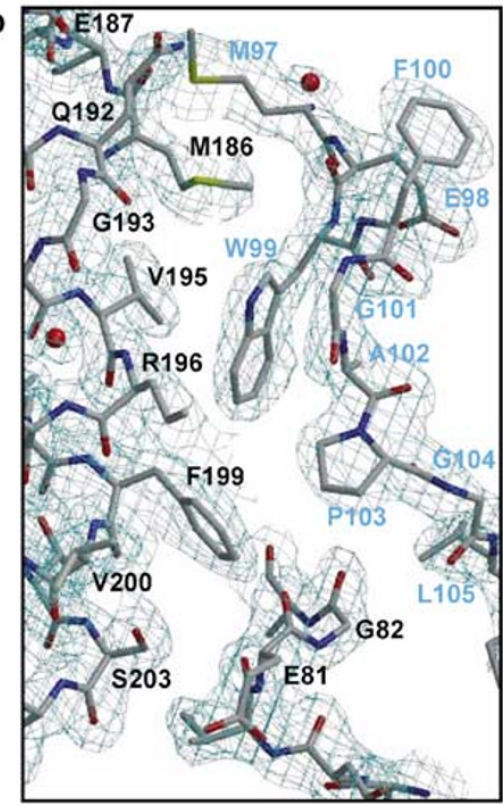

c

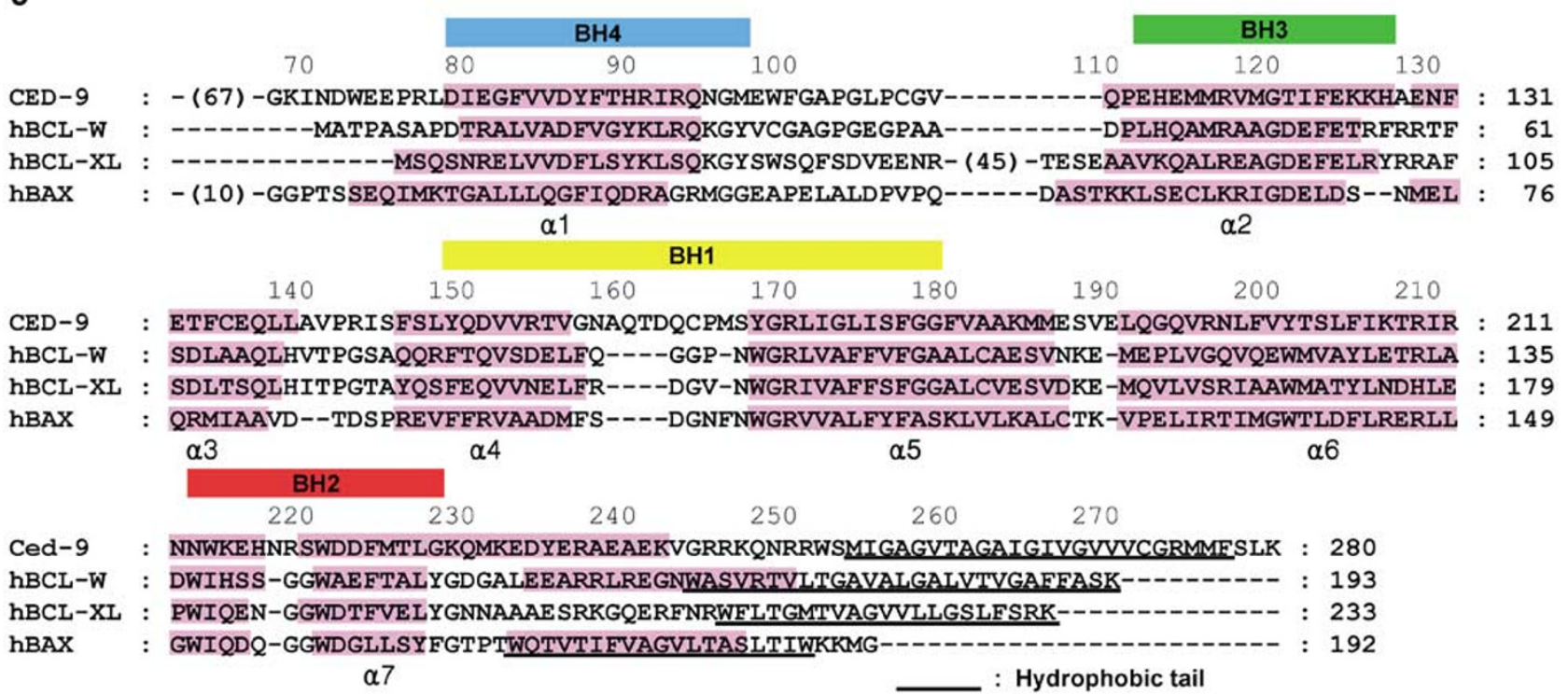

Figure 1 CED-9 structure and sequence alignment. (a) Structure of CED-9. The secondary structures are numbered in the order of appearance in the primary sequence. BH1-4 domains are color coded as indicated in (c). (b) $2 F_{0}-F_{c}$ map $(2.03 \AA, 1.1 \sigma)$ showing the region around loop $\alpha 1-\alpha 2$ with a focus on its interactions with helix $\alpha 6$. The interactions are mostly hydrophobic. The residues of the loop are labeled with blue letters. The red spheres are water molecules. (c) Structure-based sequence alignment of four different antiapoptotic BCL-2 family members. Numbers in the parentheses indicate the omitted amino acids. The $\alpha$-helices are shown in pink and the underlines indicate the C-terminal hydrophobic tails 
a
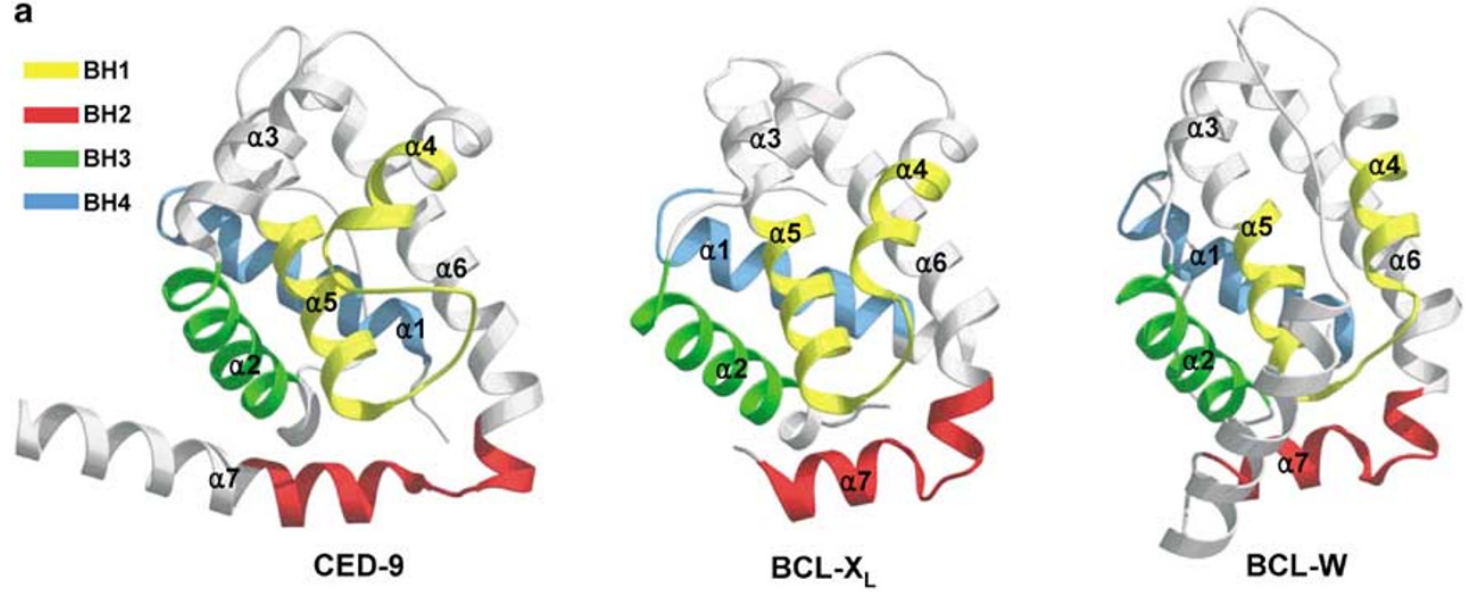

b

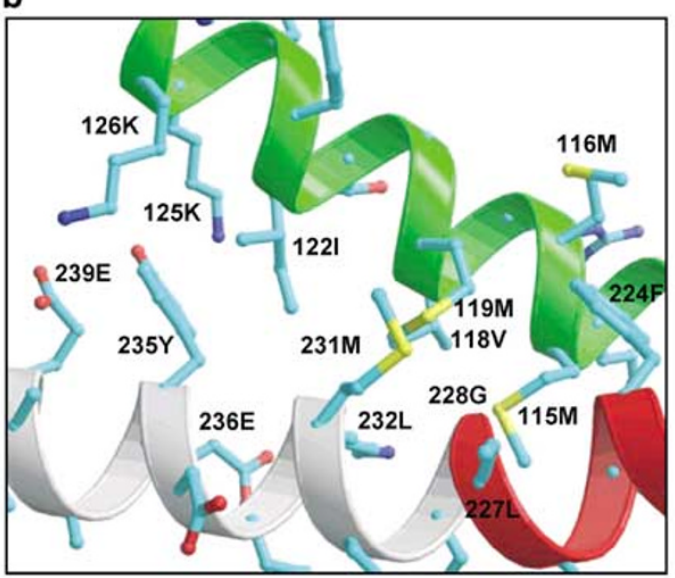

c
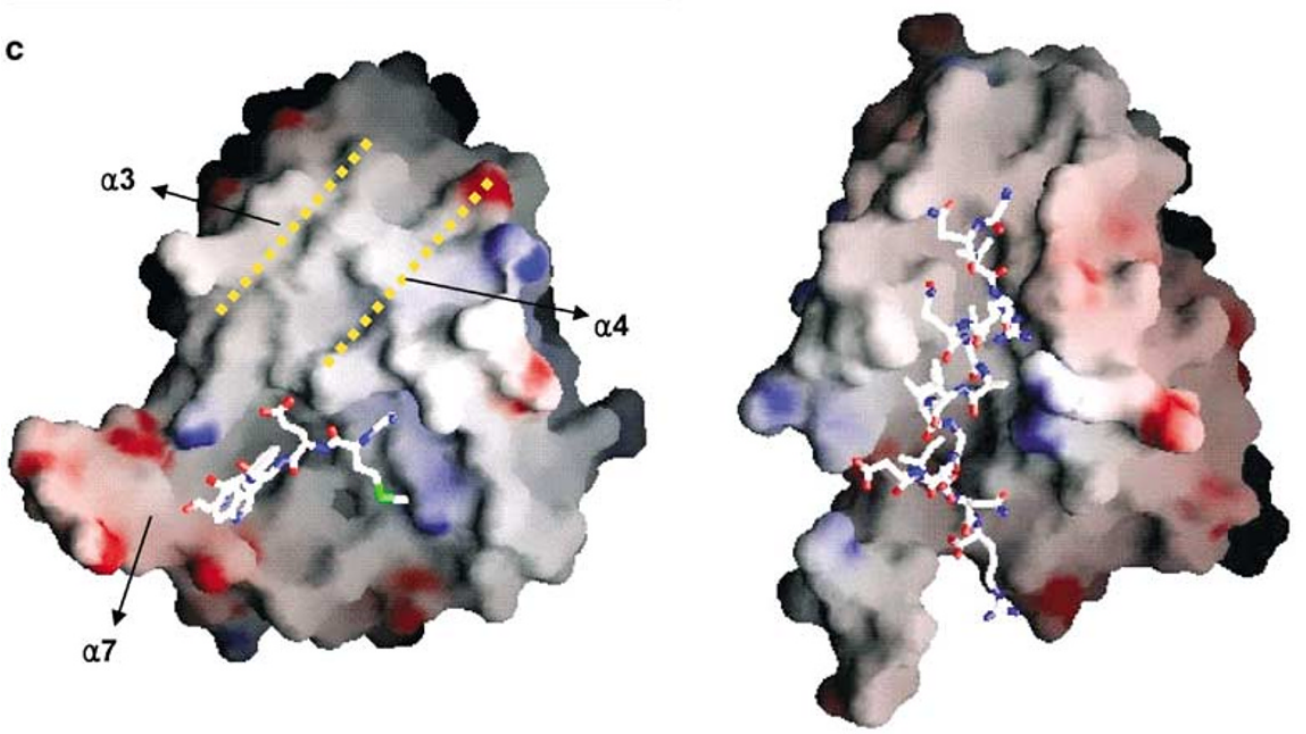

Figure 2 Unique structural features of CED-9. (a) The ribbon diagrams of CED-9 (left), BCL-X (middle, PDB code: 1MAZ), and BCL-W (right, PDB code: 1001). The structures of the C-terminal region of the three proteins (in the same orientation) are markedly different from each other. In the structures of CED-9 and BCL- $X_{L}, C$ terminal 9 and 3 residues, respectively, are disordered and absent from the structures. (b) Interactions between the C-terminal helix $\alpha 7$ (in gray and red) and $\alpha 2$ (BH3 domain, in green). The interactions are mostly hydrophobic. (c) Surface representation of CED-9 (left) and BCL-X $X_{L}$ : BAK peptide (PDB code: 1BXL) (right). The stretch of amino acids (residues 96-101), occupying the hydrophobic groove in CED-9 by crystal packing interactions, and the BAK peptide bound to BCL-X $X_{L}$ are shown in balland-sticks. The orientations of CED-9 and the BCL- $X_{L}: B A K$ peptide complex are the same and roughly the same as that of CED-9 in (a). The dotted lines indicate the axis of the helices $\alpha 3$ and $\alpha 4$ 


\section{CED-9 and EGL-1 forms an extremely stable complex}

It was observed that EGL-1 expression is dependent on the presence of CED-9 in human cells and in in vitro translation. ${ }^{26}$ Similarly, we could not detect the expression of EGL-1 alone in E. coli, but observed that the EGL-1 protein coexpressed with CED-9 from a bicistronic vector, all in the soluble fraction of the cell lysate. Several lines of observations indicate that the complex between the two proteins is extremely stable. First, the complex behaves as if it is a single protein throughout the protein purification steps and on native polyacrylamide gels (Figure 3 ). Next, the complex did not dissociate even in the presence of $5 \%$ detergent triton $\mathrm{X}-100,4 \mathrm{M} \mathrm{NaCl}$, or $1 \%$ Triton X-100 plus $1 \mathrm{M} \mathrm{NaCl}$ (Figure 3a). Finally, the melting temperature $\left(T_{M}\right)$ of the CED-9: EGL-1 complex is $74^{\circ} \mathrm{C}$, while that of CED- 9 is $49^{\circ} \mathrm{C}$. A single phase melting curve indicates

a
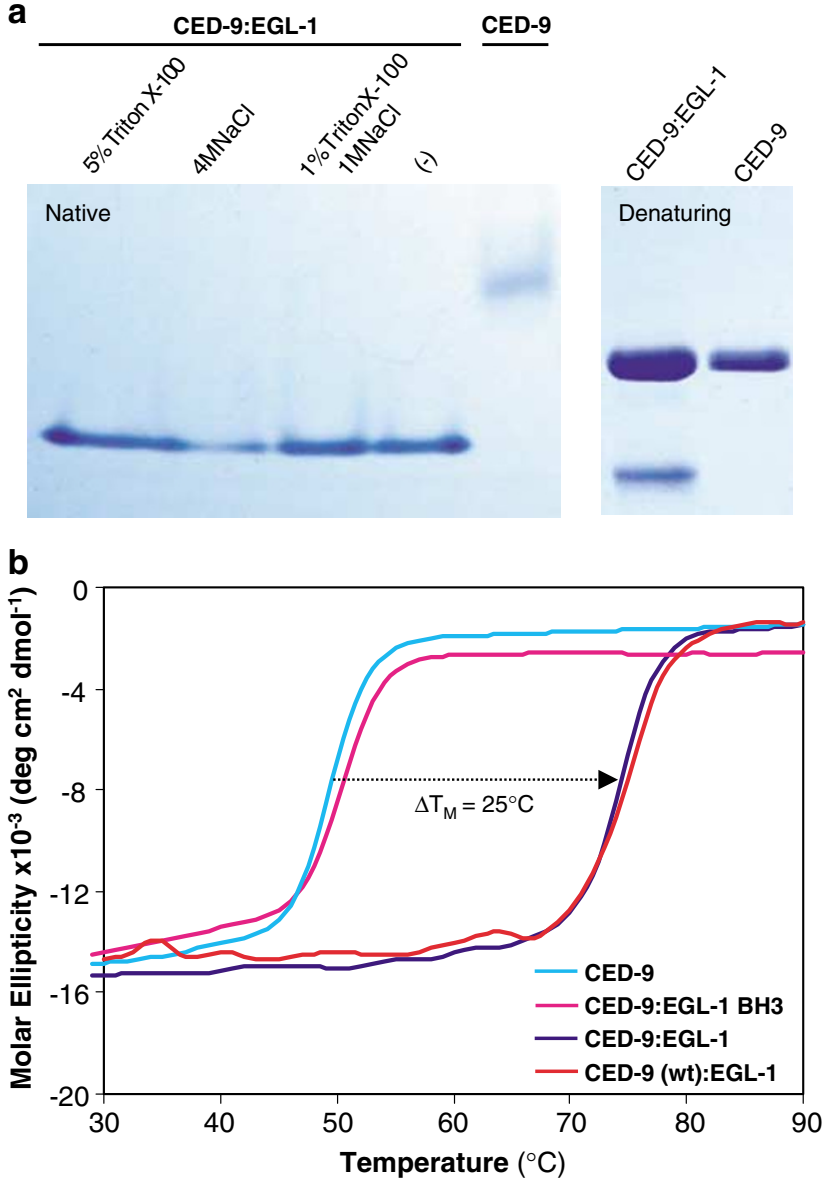

Figure 3 Extremely stable CED-9:EGL-1 complex. (a) Electrophoresis to assess the interaction between CED-9 and EGL-1. The purified CED-9: EGL-1 complex was treated for $3 \mathrm{~h}$ at room temperature under the indicated conditions and subjected to native gel electrophoresis (left). The complex does not dissociate under these conditions. The mobility of the complex, migrating as a single band on the gel, is greater than that of CED- 9 because of the acidic nature of EGL-1. Denaturing gel electrophoresis shows the homogeneity of the complex and CED-9 samples (right). (b) The effect of EGL-1 and the EGL-1 BH3 peptide on the thermal inactivation of CED-9. The peptide was mixed with the CED-9 sample (approximately 3:1 molar ratio of the peptide and CED-9) and incubated overnight at $4{ }^{\circ} \mathrm{C}$ prior to the melting temperature analysis by $\mathrm{CD}$ spectroscopy. The label 'CED-9 (wt)' indicates CED-9 (residues 68-251, no mutation) a simultaneous denaturation of CED-9 and EGL-1 (Figure 3b). It could be imagined that the $T_{\mathrm{M}}$ of $E G L-1$ is close to $74^{\circ} \mathrm{C}$, and when complexed with CED-9, it prevents the thermal denaturation of CED-9 up to this temperature. This is not the case, because CED-9 mutants in complex with EGL-1 exhibit lower $T_{M}$ 's as described below. In order to find out whether the wild-type CED-9 (residues 68-251, no mutation) forms a more stable complex with EGL-1, the wild-type CED-9 in complex with EGL-1 was produced and subjected to the $T_{M}$ analysis. As shown in Figure $3 \mathrm{~b}$, the melting curve of the wildtype complex is virtually indistinguishable from that of the CED-9 (residues 68-251, C107S/C135A/C164S): EGL-1 complex, indicating that the surface-exposed Cys107 and Cys164 do not participate in the binding interactions.

\section{Comparison of CED-9 : EGL-1 and CED-9 : EGL-1 peptide complexes by NMR and CD}

We found that thermally denatured EGL-1, which precipitated together with CED-9, could be partly recovered in the form of the CED-9: EGL-1 complex by the addition of intact CED-9. This is a manifestation of the chaperone activity of CED-9 for the folding of EGL-1. The newly formed complex is identical to that preformed in E. coli, as indicated by the identical circular dichroism (CD) spectra (Figure 4), the same mobility on a native gel, and the indistinguishable $T_{\mathrm{M}}$ values of the two (data not shown). This finding enabled us to prepare ${ }^{15} \mathrm{~N}$-labeled CED-9 in complex with unlabeled EGL-1 and to record the ${ }^{1} \mathrm{H}-$ ${ }^{15} \mathrm{~N}$ heteronuclear single-quantum (HSQC) NMR spectrum of the $\left[{ }^{15} \mathrm{~N}\right] \mathrm{CED}-9$ : EGL-1 complex. We also carried out the same NMR experiment on $\left[{ }^{15} \mathrm{~N}\right] \mathrm{CED}-9$ in the presence of three-fold molar excess of the 16 amino-acid peptide of the $\mathrm{BH} 3$ domain of EGL-1. As shown in Figure 4, the NMR spectrum of the $\left[{ }^{15} \mathrm{~N}\right] \mathrm{CED}-9$ : EGL-1 complex is drastically different from that of $\left[{ }^{15} \mathrm{~N}\right] \mathrm{CED}-9$ in the presence of EGL-1 $\mathrm{BH} 3$ peptide. The comparison demonstrates that CED-9 interacts not only with the $\mathrm{BH} 3$ domain but also with other regions of $E G L-1$. We qualitatively estimated the contribution of the $\mathrm{BH} 3$ domain of $E G L-1$ to the interaction between CED-9 and EGL- 1 by measuring the $T_{M}$ of CED- 9 in the presence of the EGL-1 BH3 peptide. In sharp contrast with EGL-1, the $\mathrm{BH} 3$ peptide increased the $T_{\mathrm{M}}$ only slightly (Figure $3 \mathrm{~b}$ ). The data also suggest that the $\mathrm{BH} 3$ domain of EGL-1 is not the sole determinant in binding to CED-9 and the contribution of this domain to the total binding affinity is not a predominant one. In order to estimate the competency of the $\mathrm{BH} 3$ peptide for replacing EGL-1 bound to CED-9, we recorded the melting temperature curve for CED-9: EGL-1 after $3 \mathrm{~h}$ of incubation at room temperature with a 30 -fold molar excess of the $\mathrm{BH} 3$ peptide. Only a slight lowering of the $T_{\mathrm{M}}\left(\sim 1^{\circ} \mathrm{C}\right)$ compared with the $T_{M}$ of CED-9: EGL-1 was observed (data not shown), confirming that the CED-9 in complex with EGL-1 is much more stable than CED-9 in complex with the $\mathrm{BH} 3$ peptide.

\section{The EGL-1-binding surface reaches the BH4 domain}

Extensive efforts to crystallize the CED-9:EGL-1 complex have been unsuccessful. Instead, we resorted to structure- 

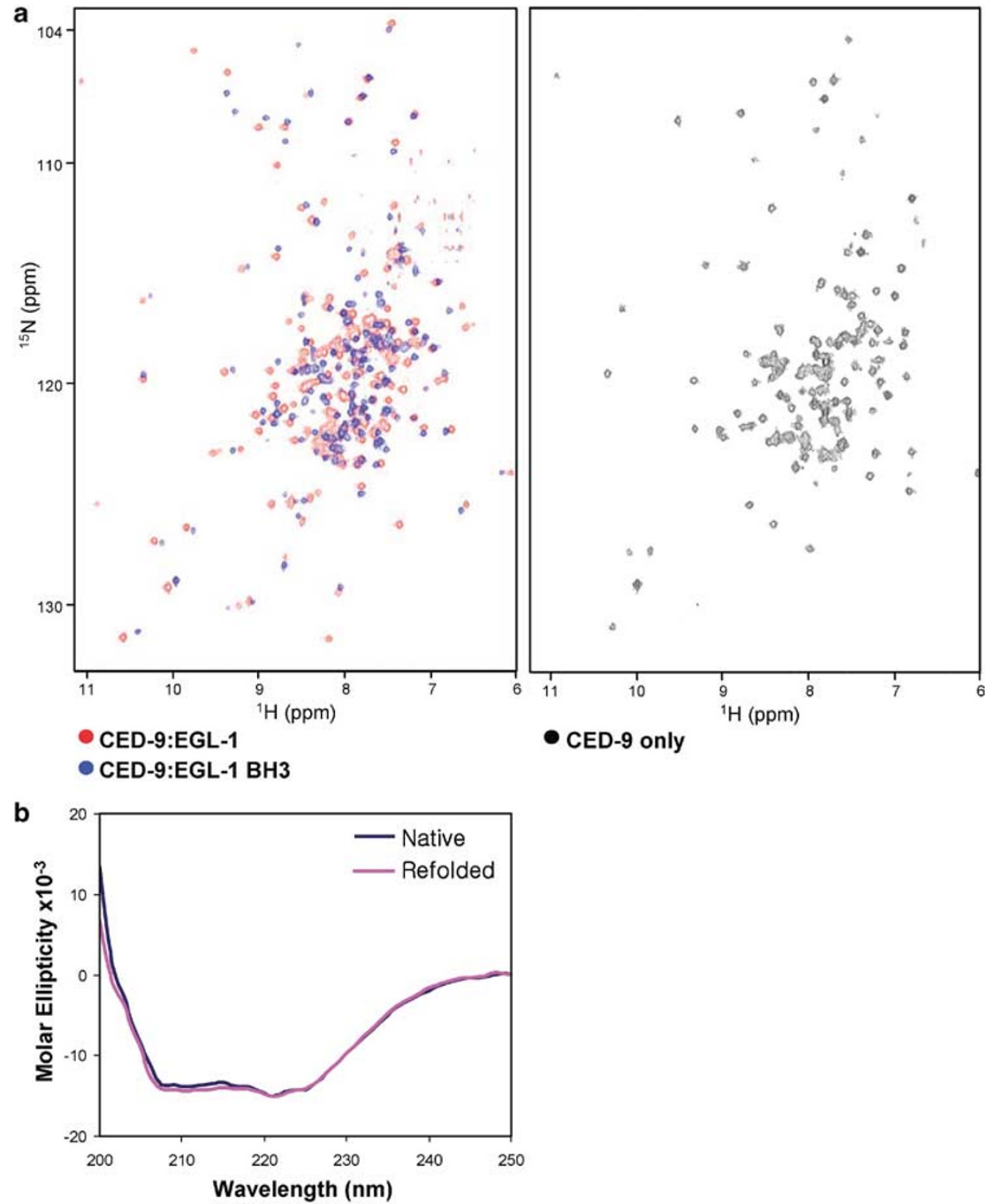

Figure 4 Binding of EGL-1 and the EGL-1 BH3 peptide to CED-9 accessed by NMR. (a) ${ }^{1} \mathrm{H}-{ }^{15} \mathrm{~N}-\mathrm{HSQC}$ spectra. The spectrum of the $\left[{ }^{15} \mathrm{~N}\right] \mathrm{CED}-9$ : EGL-1 complex (red) and the ${ }^{15} \mathrm{NJCED}-9$ in the presence of the EGL-1 BH3 peptide (blue) are superimposed (left). The concentration of CED-9 in each sample was $0.6 \mathrm{mM}$. The spectrum of $\left[{ }^{15} \mathrm{~N}\right] \mathrm{CED}-9$ alone (right) was collected at $0.3 \mathrm{mM}$ concentration of the sample. At $0.6 \mathrm{mM}$, we observed a typical pattern of broad and clumped peaks from soluble aggregates. This property of CED-9 disappeared when bound to the peptide or EGL-1. (b) CD spectra of CED-9: EGL-1 complexes. 'Native': the CED-9 : EGL-1 complex coexpressed and directly purified from E. coli cells. 'Refolded': the [ $\left.{ }^{15} \mathrm{~N}\right] \mathrm{CED}-9$ : EGL-1 complex obtained by CED-9-induced refolding of EGL-1

based mutagenesis of CED-9 followed by the analysis of $T_{\mathrm{M}}$ 's of the resulting mutants in complex with EGL-1 to map the EGL-1-binding surface on CED-9. Three CED-9 mutants were constructed, each carrying a mutation of either H90W, A128Y, or A183Y (Figure 5) in addition to the triple mutations of C107S/C135A/C164S. The three residues are at least partly exposed on the surface of CED-9, which we expected would be buried by EGL-1, if the binding of EGL-1 extends beyond the $\mathrm{BH} 3$-binding groove toward the solvent-exposed part of the $\mathrm{BH} 4$ region. A simple modeling experiment indicated that each of the substitutions does not cause a steric hindrance at all. The substitutions of these residues with the bulky residues could interfere with the binding of EGL-1, if they are indeed on the binding surface. Like the CED-9: EGL-1 complex, each of the three CED-9 mutants was coexpressed and purified as a complex with EGL-1. The CD spectra of the three CED-9 mutants in complex with EGL-1 were nearly identical to that of the CED-9: EGL-1 complex (data not shown), indicating that the mutations have no adverse effect on the structure of CED9. The $T_{M}$ of CED-9(H9OW) : EGL-1 was slightly higher than that of CED-9:EGL-1. The $T_{M}$ 's of CED-9(A128Y):EGL-1 and CED-9(A183Y):EGL-1 were slightly lower and by 
a

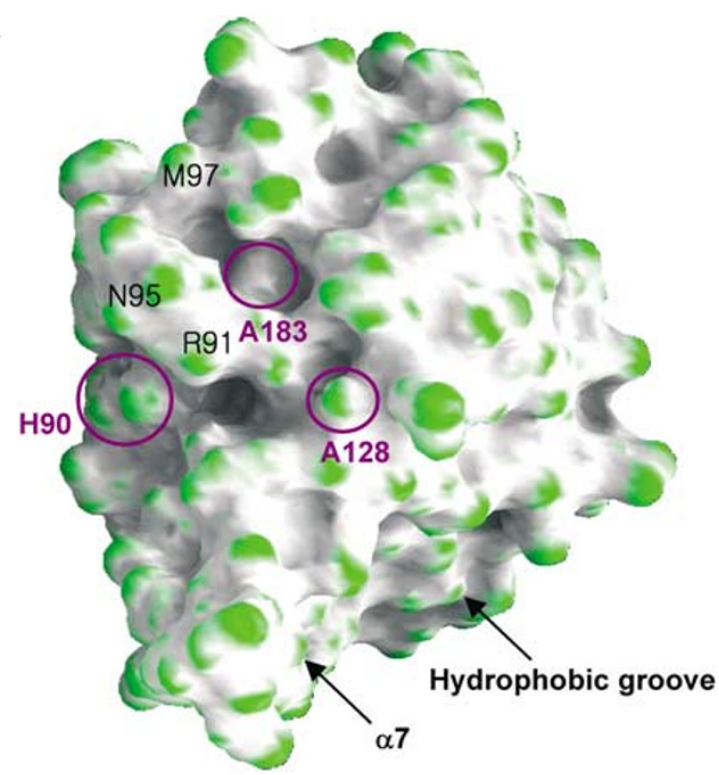

b

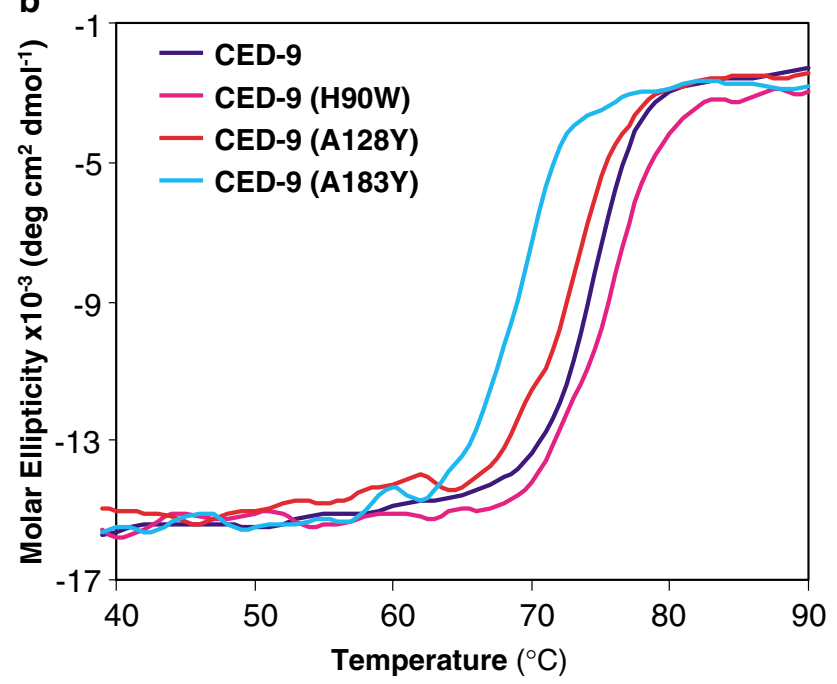

Figure 5 Point mutations of CED-9 and melting temperature analysis of mutant CED-9 : EGL-1 complexes. (a) Three different point mutations. The circles on the surface of CED-9 indicate the positions of the mutations. The three residues on the BH4 domain that surround Ala183 are labeled. (b) Melting temperature analysis by CD spectroscopy. The CED-9(A183Y): EGL-1 complex exhibits a decrease in the $T_{\mathrm{M}}$ by $5^{\circ} \mathrm{C}$, while the other two mutant complexes exhibit less than $1.5^{\circ} \mathrm{C}$ difference, compared to the CED-9:EGL-1 complex. Three independent measurements of the $T_{\mathrm{M}}$ 's indicated less than $0.5^{\circ} \mathrm{C}$ variation. It is noted that the mutation points of $\mathrm{C} 107 \mathrm{~S}$ and $\mathrm{C} 164 \mathrm{~S}$ are located at the back of the surface shown in (a)

$5^{\circ} \mathrm{C}$, respectively, compared with that of CED-9:EGL-1 (Figure 5b). Ala128 is located at the beginning end of $\alpha 3$ and Ala183 is located in the middle of a pocket (Figure $5 \mathrm{a}$ ), which is lined by residues on the BH4 domain (Arg91, Asn95, Met97) and on $\alpha 5$ (Phe180, Lys184, Glu187). The substantial decrease in the thermal stability of the A183Y mutant indicates that the binding interface for EGL-1 extends beyond the BH3-binding groove of CED-9 and reaches at least a part of the $\mathrm{BH} 4$ domain.

\section{Discussion}

The study presented here reveals how the entire CED-9 molecule is structurally organized. The molecule has an unstructured part (residues 1-67), the structured BCL-2 homology domain (residues 68-242), a flexible linker (243251), and the putative transmembrane region (residues 252-280). The C-terminal segments of the proapoptotic BCL-2 family members are nonuniform in length and bear limited sequence homologies. The structure of CED-9, together with those of the other BCL-2-like molecules, reveals the structural dissimilarity of the $\mathrm{C}$-terminal segments in these proteins, which should be directly related with their functional roles. By forming a prominent hydrophobic groove at the $\mathrm{BH} 3-$ binding region, the functional role of the helix $\alpha 7$ (residues 220-242) of CED-9 is likely to lie in the recognition of EGL-1 (and CED-4) rather than in the regulation of the interactions with the partner proteins. While mammalian BCL-2-like proteins interact with many different partner proteins, CED-9 is known to interact only with the CED-4 and EGL-1. Perhaps, regulating the ligand access to the binding groove by the $\mathrm{C}$ terminal segment, which was observed for BCL-W and BAX, is not necessary for CED-9.

We demonstrated that regions other than the BH3-binding groove of CED-9 and the $\mathrm{BH} 3$ domain of EGL-1 are extensively involved in the interactions between CED-9 and EGL-1 to form an extremely stable complex. This binding interaction should be the basis for the onset of apoptosis in $C$. elegance by releasing CED-4 from CED-9. While the $\mathrm{BH} 3$ domains are 9-16 amino-acid stretches, most of the BH3-only proteins have more than 100 amino acids. We speculate that the $\mathrm{BH} 3$ domain is the common interaction motif, but other surface regions of probably many of the $\mathrm{BH} 3-$ only proteins contribute significantly to the binding affinity for the antiapoptotic BCL-2-like molecules. Indirect observations supporting this idea are found in the literature. The dissociation constant for the binding between the $\mathrm{BH} 3$ peptide of NOXA and BCL-2 is greater than $1 \mu \mathrm{M} .{ }^{16}$ Considering that NOXA is a transcriptionally regulated potent inducer of apoptosis, like EGL-1, the dissociation constant between the full-length NOXA and BCL-2 would be significantly lower than $1 \mu \mathrm{M}$. Another example is the interaction between $B C L-X_{L}$ and $B I M$, where deamidation of two conserved asparagine residues on the unstructured loop $\alpha 1-\alpha 2$ of $B C L-X_{L}$ reduces the binding of BIM to the protein. ${ }^{27}$ It is hard to imagine that the deamidation at the unstructured loop would affect the binding of BIM by causing some tertiary structural change at the BH3-binding groove of $\mathrm{BCL}-\mathrm{X}_{\mathrm{L}}$. A plausible explanation would be that BIM interacts with loop $\alpha 1-\alpha 2$ of BCL-X $X_{L}$, and the deamidation on this loop causes charge repulsion between the two proteins. It has been suggested that the binding specificity and the hierarchy of binding affinity between the mammalian BCL-2 family members could be the basis of the action mechanism of these proteins. ${ }^{16}$ Elucidation of the full pictures of several complexes between the antiapoptotic and the BH3-only members will provide excellent frameworks leading to a higher level of understanding of the intricate interplay between the BCL-2 family members. 


\section{Materials and Methods}

\section{Plasmid construction, protein expression, and purification}

The full-length CED-9 was amplified by polymerase chain reaction (PCR) from the $C$. elegans RB1 cDNA library. The PCR products were cloned into the pRSET-A vector (Invitrogen). Subsequently, the CED-9 DNA fragment coding for residues 64-251 was cloned into the same sites of pRSET-A vector. The EGL-1 gene including a single intron between two exons was amplified by PCR from a $C$. elegans genomic DNA. The PCR products were cloned into the $\mathrm{PET} 21 \mathrm{~d}$ vector (Novagen). The intron was removed from the vector by a PCR technique followed by a blunt-end ligation after polynucleotide kinase treatment. Subsequently, the DNA fragment encoding the ribosome-binding site and EGL-1 was inserted into the pRSET-A vector that carried the CED-9 gene. The bicistronic vector was transformed into the $E$. coli BL21 (DE3) pLysS strain. From this vector, CED-9 was produced with a (His) ${ }_{6}$-tag at the $\mathrm{N}$-terminus, while EGL-1 was produced without a tag. The expression of the two proteins was induced by $0.67 \mathrm{mM}$ isopropyl-D-thiogalactopyranoside at an optical density of 1.0 at $298 \mathrm{~K}$ for $8 \mathrm{~h}$. The expression level of CED-9 was about five times higher than that of $E G L-1$. Bacterial lysate was prepared by sonication in buffer $A$ consisting of $20 \mathrm{mM}$ sodium phosphate ( $\mathrm{pH} 7.2), 100 \mathrm{mM} \mathrm{NaCl}$, and $2 \mathrm{mM}$ $\beta$-mercaptoethanol. CED-9 and the CED-9: EGL-1 complex bound to a Ni-NTA column (QIAGEN) were eluted with buffer A containing $200 \mathrm{mM}$ imidazole. The eluted fractions were pooled and diluted four-fold with buffer B consisting of $20 \mathrm{mM}$ sodium phosphate, $50 \mathrm{mM} \mathrm{NaCl}$, and $2 \mathrm{mM}$ $\beta$-mercaptoethanol. The protein sample was loaded onto HitrapQ column (Amercham Pharmacia) pre-equilibrated with buffer B. CED-9 and the CED-9 : EGL-1 complex were eluted at $\mathrm{NaCl}$ concentrations of 100-150 and $200-250 \mathrm{mM}$, respectively. The (His) ${ }_{6}$-tag of CED-9 was removed by recombinant human caspase-3 (prepared in house) that readily hydrolyzed the peptide bond between Asp67 and Gly68, a site cleaved by CED-3. ${ }^{18}$ The reaction mixtures were separately loaded onto a Ni-NTA column to remove caspase- 3 that bound to the column due to a (His) $)_{6}$-tag at the $\mathrm{N}$-terminus of the enzyme. The unbound fraction was loaded onto a HitrapQ column again. CED-9 did not bind to the column, while the complex was eluted at the $\mathrm{NaCl}$ concentration of $150-200 \mathrm{mM}$. The purified protein samples were dialyzed against buffer $B$ and then concentrated to $10 \mathrm{mg} / \mathrm{ml}$ using a Vivaspin 20 (Satorius). Each of the $\mathrm{H} 90 \mathrm{~W}, \mathrm{~A} 128 \mathrm{Y}$, and $\mathrm{A} 183 \mathrm{Y}$ mutations were introduced to the CED-9 gene construct that contained the triple mutations of $\mathrm{C} 107 \mathrm{~S} / \mathrm{C} 135 \mathrm{~A} / \mathrm{C} 164 \mathrm{~S}$ by using QuikChange Kit (Stratagene), and each mutant in complex with EGL-1 was purified according to the same purification procedures for the CED-9 : EGL-1 complex.

\section{Crystallization and structure determination}

Crystals of the CED-9 were obtained by the hanging-drop vapor-diffusion method at $285 \mathrm{~K}$ with the reservoir solution composed of $10 \%$ polyethylene glycol 8000 and $0.1 \mathrm{M} \mathrm{HEPES}(\mathrm{pH} 7.5)$ at $12^{\circ} \mathrm{C}$. Before data collection,

Table 1 Data collection and structure solution

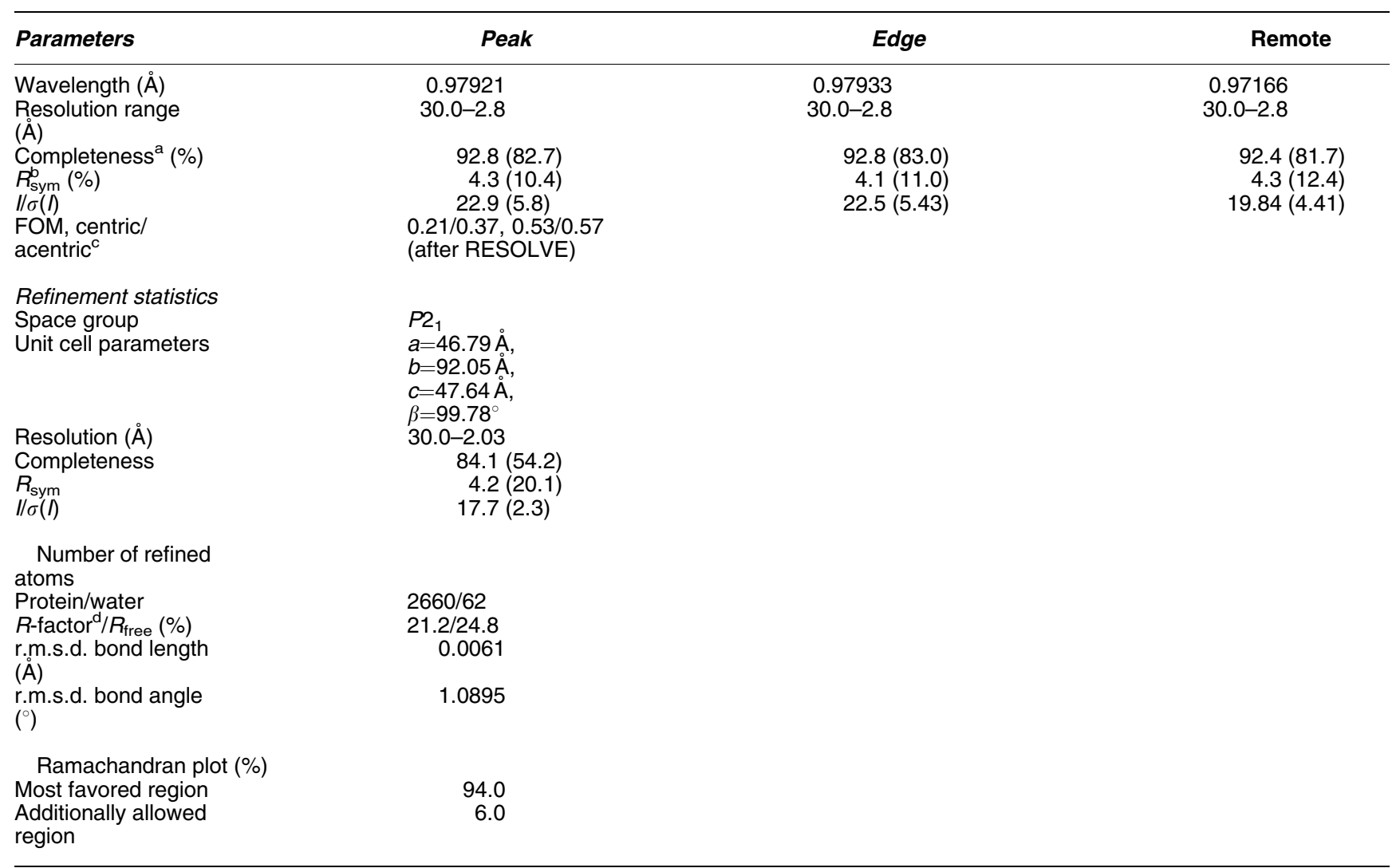

${ }^{\mathrm{a}}$ Numbers in parentheses are statistics from the highest resolution shell

${ }^{\mathrm{b}} R_{\text {sym }}=\Sigma I_{\text {obs }}-I_{\text {avg }} / I_{\text {obs }}$, where $I_{\text {obs }}$ is the observed intensity of individual reflection and $I_{\text {avg }}$ is the average over symmetry equivalents ${ }^{\mathrm{c}}$ Figure of merit, defined as $<$ $\left|\Sigma P(\alpha) \mathrm{e}^{\mathrm{i} \alpha} / \Sigma P(\alpha)\right\rangle$, where $\alpha$ is the phase and $P(\alpha)$ is the phase probability distribution ${ }^{\mathrm{d}} R$-factor $=\Sigma|| F_{\mathrm{o}}|-| F_{\mathrm{c}}|\Sigma| F_{\mathrm{o}} \mid$, where $\left|F_{\mathrm{o}}\right|$ and $\left|F_{\mathrm{c}}\right|$ are the observed and calculated structure factor amplitudes, respectively. $R_{\text {free }}$ was calculated with $5 \%$ of the data 
crystals of CED-9 were immersed briefly in a cryoprotectant solution, which is the precipitant solution containing $20 \%$ glycerol. A MAD data set at three different wavelengths was collected with a crystal of selenomethionine-substituted CED-9 using synchrotron radiation from the beamline $6 \mathrm{~B}$ at Pohang Accelerator Laboratory, Korea, and processed using the programs DENZO and SCALEPACK. ${ }^{28}$ Phase determination was carried out at $2.8 \AA$ with the program SOLVE, ${ }^{29}$ and phases were subsequently improved by density modification with the program RESOLVE. ${ }^{30}$ The electron density was of high quality showing nearly all features of protein side chains. The program $\mathrm{O}^{31}$ was used for model building. The $2.03 \AA$ data were collected later using a crystal of selenomethionine-substituted CED-9 and used for the final refinement performed with the CNS package. ${ }^{32}$ The two molecules in the asymmetric unit superpose onto each other with an R.M.S.D. value of $0.93 \AA$. Crystallographic data statistics are summarized in Table 1.

\section{CD spectroscopy}

Data were collected on a JASCO J-715 spectropolarimeter equipped with a thermoelectric temperature controller. $C D$ spectra were recorded with protein samples $(2.5-10 \mu \mathrm{M})$ in $20 \mathrm{mM}$ phosphate buffer $(\mathrm{pH} 7.5)$ at $25^{\circ} \mathrm{C}$ over the range of $200-250 \mathrm{~nm}$ in a nitrogen atmosphere. Each spectrum is the accumulation of three scans corrected by subtracting signals from the buffer control. Thermal denaturation was performed by heating protein samples $(11.5-20 \mu \mathrm{M})$ at a constant rate of $0.5^{\circ} \mathrm{C} / \mathrm{min}$, while monitoring the ellipticity at $222 \mathrm{~nm}$. $T_{M}$ is defined as the temperature at which $50 \%$ of protein molecules are unfolded.

\section{EGL-1 BH3 peptide}

The 16 amino acids peptide, corresponding to residues $52-67$ of EGL-1 and with a C-terminal modification, ${ }^{+} \mathrm{H}_{3} \mathrm{~N}-\mathrm{Y}^{52}$ EIGSKLAAMCDDFDA ${ }^{67}$. $\mathrm{CONH}_{2}$, was purchased from Anygen, Inc. (Korea) and used without further purification. The sample was at least $98 \%$ pure and the molecular mass of the peptide was confirmed by mass spectrometry.

\section{Preparation of the NMR sample}

${ }^{15} \mathrm{~N}$-labeled CED-9 was produced from $E$. coli cells grown on M9 media supplemented with ${ }^{15} \mathrm{~N}$ ammonium chloride. The labeled sample was purified according to the same protocol for the purification of unlabeled CED-9. The $\left[{ }^{15} \mathrm{~N}\right] \mathrm{CED}-9$ in complex with unlabeled EGL-1 was produced by adding $\left[{ }^{15} \mathrm{~N}\right] \mathrm{CED}-9$ to the thermally denatured CED-9 : EGL-1 complex. The unlabeled complex was diluted to a concentration of $0.25 \mathrm{mg} / \mathrm{ml}$ in a buffer solution containing $100 \mathrm{mM}$ Tris- $\mathrm{HCl}(\mathrm{pH} 7.5), 5 \mathrm{mM} \beta$-mercaptoethanol, and $50 \mathrm{mM} \mathrm{NaCl}$. The sample was incubated for $25 \mathrm{~min}$ at $77^{\circ} \mathrm{C}$ and cooled down at room temperature. The ${ }^{15} \mathrm{~N}$-labeled CED- 9 was added to the denatured sample at a molar ratio of $1: 2$ (intact CED- 9 : denatured CED-9). After incubation for $30 \mathrm{~min}$, the mixture was centrifuged and the supernatant was loaded onto a Hitrap $Q$ column. The eluted fraction contained the $\left[{ }^{15} \mathrm{~N}\right] \mathrm{CED}-9$ : EGL- 1 complex. About $25 \%$ of added $\left[{ }^{15} \mathrm{~N}\right] \mathrm{CED}-9$ was lost.

\section{NMR spectroscopy}

${ }^{1} \mathrm{H}-{ }^{15} \mathrm{~N}-\mathrm{HSQC}$ spectra were recorded at $25^{\circ} \mathrm{C}$ on a Bruker DRX500 spectrometer equipped with a triple resonance probe and pulsed field gradients. A total of 2048 and 256 data points were collected in the $t_{2}$ and $t_{1}$ domains, respectively. The spectral widths for each time domain and the number of scan were $7002.8 \mathrm{~Hz}\left(t_{2}\right), 1520.2 \mathrm{~Hz}\left(t_{1}\right)$, and 32 or 128 scans, respectively. Spectra were processed using the XWINNMR software (Bruker AG, Germany).

\section{Coordinates}

The coordinates of the CED-9 structure have been deposited in the Protein Data Bank under the accession codes $1 \mathrm{OHU}$ with the condition of immediate release upon publication.

\section{Acknowledgements}

We thank Professors J Ahnn (KJIST) and J Lee (Yonsei University) for providing the $C$. elegance $\mathrm{CDNA}$ library. This study used the beamline $6 \mathrm{~B}$ at the Pohang Accelerator Laboratory and was supported by Creative Research Initiatives (to B-HO) and by the National Research Laboratory program (M1-0203-00-0020) (to WL) of the Korean Ministry of Science and Technology. J-SW, J-SJ, and $\mathrm{N}-\mathrm{CH}$ were supported by the Brain Korea 21 Project.

\section{References}

1. Adams JM and Cory S (2001) Life-or-death decisions by the Bcl-2 protein family. Trends Biochem. Sci. 26: 61-66

2. Puthalakath $H$ and Strasser $A$ (2002) Keeping killers on a tight leash: transcriptional and post-translational control of the pro-apoptotic activity of BH3-only proteins. Cell Death Differ. 9: 505-512

3. Bouillet $P$ and Strasser A (2002) BH3-only proteins - evolutionarily conserved proapoptotic Bcl-2 family members essential for initiating programmed cell death. J. Cell. Sci. 115: 1567-1574

4. Metzstein MM, Stanfield GM and Horvitz HR (1998) Genetics of programmed cell death in C.elegans: past, present and future. Trends Genet. 14: 410-416

5. Zou H, Henzel WJ, Liu X, Lutschg A and Wang X (1997) Apaf-1, a human protein homologous to $C$. elegans CED-4, participates in cytochrome $c$ dependent activation of caspase-3. Cell 90: 405-413

6. Yang X, Chang HY and Baltimore D (1998) Essential role of CED-4 oligomerization in CED-3 activation and apoptosis. Science 281: 1355-1357

7. Chinnaiyan AM, O'Rourke K, Lane BR and Dixit VM (1997) Interaction of CED4 with CED-3 and CED-9: a molecular framework for cell death. Science 275 : $1122-1126$

8. Spector MS, Desnoyers S, Hoeppner DJ and Hengartner MO (1997) Interaction between the $C$. elegans cell-death regulators CED-9 and CED-4. Nature 385: 653-656

9. Wu D, Wallen HD, Inohara N and Nunez G (1997) Interaction and regulation of the Caenorhabditis elegans death protease CED-3 by CED-4 and CED-9. J. Biol. Chem. 272: 21449-21454

10. Wu D, Wallen HD and Nunez G (1997) Interaction and regulation of subcellular localization of CED-4 by CED-9. Science 275: 1126-1129

11. Hengartner MO (1999) Programmed cell death in the nematode $C$. elegans Recent. Recent Prog. Horm. Res. 54: 213-222

12. Chen F, Hersh BM, Conradt B, Zhou Z, Riemer D, Gruenbaum $Y$ and Horvitz HR (2000) Translocation of $C$. elegans CED-4 to nuclear membranes during programmed cell death. Science 287: 1485-1489

13. del Peso L, Gonzalez VM, Inohara N, Ellis RE and Nunez G (2000) Disruption of the CED-9.CED-4 complex by EGL-1 is a critical step for programmed cell death in Caenorhabditis elegans. J. Biol. Chem. 275: 27205-27211

14. Chittenden T, Flemington $C$, Houghton $A B$, Ebb RG, Gallo GJ, Elangovan $B$, Chinnadurai $G$ and Lutz RJ (1995) A conserved domain in Bak, distinct from $\mathrm{BH} 1$ and $\mathrm{BH} 2$, mediates cell death and protein binding functions. EMBO J. 14: 5589-5596

15. Wang K, Yin XM, Chao DT, Milliman CL and Korsmeyer SJ (1996) BID: a novel BH3 domain-only death agonist. Genes Dev. 10: 2859-2869

16. Letai A, Bassik MC, Walensky LD, Sorcinelli MD, Weiler S and Korsmeyer SJ (2002) Distinct BH3 domains either sensitize or activate mitochondria apoptosis, serving as prototype cancer therapeutics. Cancer Cell 2: 183-192 
17. Sattler M, Liang H, Nettesheim D, Meadows RP, Harlan JE, Eberstadt M, Yoon HS, Shuker SB, Chang BS, Minn AJ, Thomson SB and Fesik SW (1997) Structure of Bcl-xL-Bak peptide complex: recognition between regulators of apoptosis. Science 275: 983-986

18. Xue D and Horvitz HR (1997) Caenorhabditis elegans CED-9 protein is a bifunctional cell-death inhibitor. Nature 390: 305-308

19. Parrish J, Metters H, Chen L and Xue D (2000) Demonstration of the in vivo interaction of key cell death regulators by structure-based design of second-site suppressors. Proc. Natl. Acad. Sci. USA 97: 11916-11921

20. Muchmore SW, Sattler M, Liang H, Meadows RP, Harlan JE, Yoon HS, Nettesheim D, Chang BS, Thompson CB, Wong SL, Ng SL and Fesik SW (1996) X-ray and NMR structure of human Bcl-xL, an inhibitor of programmed cell death. Nature 381 : $335-341$

21. Aritomi M, Kunishima N, Inohara N, Ishibashi Y, Ohta S and Morikawa K (1997) Crystal structure of rat $\mathrm{Bcl}-\mathrm{xL}$. Implications for the function of the $\mathrm{Bcl}-2$ protein family. J. Biol. Chem. 272: 27886-27892

22. Suzuki M, Youle RJ and Tjandra N (2000) Structure of Bax: coregulation of dimer formation and intracellular localization. Cell 103: 645-654

23. Hinds MG, Lackmann M, Skea GL, Harrison PJ, Huang DC and Day CL (2003) The structure of Bcl-w reveals a role for the $\mathrm{C}$-terminal residues in modulating biological activity. EMBO J. 22: 1497-1507

24. Denisov AY, Madiraju MS, Chen G, Khadir A, Beauparlant P, Attardo G, Shore GC and Gehring K (2003) Solution structure of human BCL-w: modulation of ligand binding by the C-terminal helix. J. Biol. Chem. 21: 21
25. Petros AM, Medek A, Nettesheim DG, Kim DH, Yoon HS, Swift K, Matayoshi ED, Oltersdorf T and Fesik SW (2001) Solution structure of the antiapoptotic protein bcl-2. Proc. Natl. Acad. Sci. USA 98: 3012-3017

26. del Peso L, Gonzalez VM and Nunez G (1998) Caenorhabditis elegans EGL-1 disrupts the interaction of CED-9 with CED-4 and promotes CED-3 activation. J. Biol. Chem. 273: 33495-33500

27. Deverman BE, Cook BL, Manson SR, Niederhoff RA, Langer EM, Rosova I, Kulans LA, Fu X, Weinberg JS, Heinecke JW, Roth KA and Weintraub SJ (2002) Bcl-xL deamidation is a critical switch in the regulation of the response to DNA damage. Cell 111: 51-62

28. Otwinowski Z and Minor W (1997) Processing of X-ray diffraction data collected in oscillation mode. Methods Enzymol. 276: 307-326

29. Terwilliger TC and Berendzen J (1999) Automated MAD and MIR structure solution. Acta Crystallogr. D 55: 849-861

30. Terwilliger TC (2000) Maximum-likelihood density modification. Acta Crystallogr. D 56: 965-972

31. Jones TA, Zou JY, Cowan SW and Kjeldgaard M (1991) Improved methods for binding protein models in electron density maps and the location of errors in these models. Acta Crystallogr. A 47: 110-119

32. Brunger AT, Adams PD, Clore GM, DeLano WL, Gros P, Grosse-Kunstleve RW, Jiang JS, Kuszewski J, Nilges M, Pannu NS, Read RJ, Rice LM, Simonson T and Warren GL (1998) Crystallography \& NMR system: a new software suite for macromolecular structure determination. Acta Crystallogr. D 54: $905-921$ 